\title{
Una alternativa para algunos algoritmos algebraicos en un curso de matemáticas básicas ${ }^{1}$
}

\author{
An alternative for some algebraic algorithms on a basic math course \\ Uma alternativa para alguns algoritmos algébricos \\ sobre um curso de matemática básica
}

Recibido: mayo 2013 Aceptado: agosto 2013
Saulo Mosquera López ${ }^{2}$

\section{Resumen}

En esta presentación se ilustran procedimientos de álgebra elemental, diferentes a los usuales, en cuanto a resolución de ecuaciones con radicales y ecuaciones de segundo grado, no muy utilizados o poco conocidos por los docentes de esta área, los cuales son fruto de una experiencia con los estudiantes de primer semestre del programa de Licenciatura en Matemáticas de la Universidad de Nariño.

Palabras clave: Matemáticas escolares; álgebra; ecuaciones; algoritmos; competencias; estándares.

\section{Abstract}

This presentation will illustrate methods of elementary algebra, different from the usual, as for solving equations with radicals and quadratic equations, not used or poorly understood by teachers in this area, which are the result of experience with students of first semester degree program in mathematics from the University of Nariño.

Keywords: School mathematics, algebra, equations, algorithms, skills, standards.

\section{Resumo}

Esta apresentação irá ilustrar métodos de álgebra elementar, diferentes do habitual, como para a resolução de equações com radicais e equações quadráticas, não utilizados ou mal compreendida pelos professores nesta área, que são o resultado da experiência com alunos do primeiro programa de graduação semestre de matemática da Universidade de Nariño.

Palavras-chave: A matemática escolar, álgebra, equações, algoritmos, habilidades, normas. 


\section{Contextualización}

En diferentes programas profesionales del País, en particular en los programas de Ingeniería Civil e Ingeniería Electrónica de la Universidad de Nariño, se ha eliminado de la estructura curricular, la asignatura Matemáticas Básicas y se inicia con un curso de Cálculo Diferencial, sin embargo, en el programa de Licenciatura en Matemáticas de esta Institución, este curso se ha conservado y se desarrolla alrededor de temas que posibilitan rescatar las metas terminales de la formación matemática que es razonable esperar de un egresado de las Instituciones de Educación Media, pretende capacitar al estudiante en el análisis de los elementos teóricos y en el manejo de los procesos operatorios de la asignatura, que le permitan asimilar los conocimientos de los cursos posteriores, que son elementos fundamentales en la formación de un futuro docente.

Las consecuencias de la eliminación de esta asignatura aún no han sido evaluadas, sin embargo, un hecho real es que en la Universidad de Nariño, la mortalidad estudiantil, en los cursos de Cálculo Diferencial de los programas mencionados, oscila entre el 60 y el 70\%, lo cual, complementariamente, se considera, que está determinado por la relación entre COMPETENCIAS y CONOCIMIENTOS. En este contexto, el Ministerio de Educación Nacional (2006) declara que la enseñanza básica y media debe girar alrededor del logro de competencias, sin embargo, en la enseñanza universitaria, en general, se trabaja con base en conocimientos.

En la búsqueda de una conciliación entre estos enfoques, en el programa de Licenciatura en Matemáticas de la Universidad de Nariño, se ha intentado buscar maneras alternas para que los futuros docentes se apropien de algunos algoritmos algebraicos no usuales, que potencien determinados Estándares Básicos de Competencias (Ministerio de Educación Nacional, 2006), y en consecuencia, el propósito de la ponencia es, presentar algunos de los resultados de esta labor que se consideran adecuados para compartir con los docentes de la Educación Básica y Media de Colombia.

\section{Referentes Teórico - Prácticos Básicos}

En los Estándares Básicos de Competencias de Matemáticas, grados octavo a noveno, se encuentran, entre otros, los siguientes: "construyo expresiones algebraicas equivalentes a una expresión algebraica dada", "Identifico diferentes métodos para solucionar ecuaciones" y en los libros de texto utilizados en la Enseñanza Básica, como por ejemplo, en Chávez, Castañeda, Gómez, Joya, Chizner \& Gómez (2010), se presentan algunos métodos que permiten tratar estos estándares. Complementariamente, el Ministerio de Educación Nacional $(1998,2006)$ destaca la importancia de fomentar y desarrollar uno de los cinco procesos generales de la actividad matemática como es el de formular, comparar y ejercitar procedimientos y algoritmos. De esta manera, en esta experiencia de aula, se presentan algoritmos, alternos a los usuales, para resolver ecuaciones con radicales y ecuaciones cuadráticas en cuya práctica, los estudiantes adquirieron destrezas en su aplicación y tuvieron la posibilidad de compararlos con los algoritmos tradicionales y apreciar sus ventajas y desventajas.

En palabras de Skovsmose (2000) son dos los aspectos a considerar al caracterizar la enseñanza de las matemáticas en el aula de clase: los tipos de referencia (matemáticas puras, semirealidad y situaciones de la vida real) y la clase de escenario (paradigma del ejercicio y escenarios de investigación). Donde la bina \{Matemáticas pura, paradigma del ejercicio\} se impone como la más utilizada en las clases de matemáticas. El propósito de la presentación es poner en evidencia algoritmos desarrollados como parte del proceso de enseñanza en el desarrollo de la asignatura "Matemáticas Elementales" del primer semestre del programa de Licenciatura en Matemáticas de la Universidad de Nariño, donde en la enseñanza se privilegió la 2-upla \{Matemáticas pura, Escenarios de investigación\} a través de procesos de indagación y exploración; así, como del trabajo colaborativo. 


\section{Descripción general de la experiencia de aula}

Puesto que el propósito era proponer situaciones que generaran, a través del trabajo colaborativo, ambientes de aprendizaje que plantearan métodos alternativos que permitieran modificar o superar los algoritmos usuales, en algunas tareas algebraicas, se propusieron temas a tratar como: resolución de ecuaciones con radicales y ecuaciones de segundo grado.

Ecuaciones con radicales. Para iniciar, se planteó una actividad rutinaria como la siguiente:

Hallar, en números reales, el conjunto solución de la ecuación:

\section{$\sqrt{ }$}

En general, los estudiantes recordaban el procedimiento estándar de "elevar al cuadrado", aunque algunos de ellos lo aplicaban de manera incorrecta. Después de las aclaraciones correspondientes se pasó a la actividad central que fue:

¿Es posible hallar algoritmos, diferentes a los usuales, que permitan resolver ecuaciones similares a la anterior?

Realmente no se tuvieron respuestas cercanas a las que se esperaban por lo que se tuvo la necesidad de dirigir el trabajo con preguntas tales como:

¿Es posible transformar la ecuación dada en una ecuación cuadrática?

Después de un análisis y discusión de diferentes alternativas, se llegó por consenso al siguiente procedimiento.

Dado que dentro del radical se encuentra la expresión para obtener una ecuación cuadrática es necesario sumar a lafuera del radical, y para que la expresión no se altere se debe restar con lo cual se obtiene la ecuación: $\sqrt{ }$

Después de esto para la generalidad de los estudiantes fue posible reconocer esta igualdad como una ecuación cuadrática y obtener la solución de la misma.

Para afianzar el procedimiento descrito anteriormente se propuso la siguiente actividad.

¿Es posible aplicar, la estrategia utilizada, para hallar, en números reales, el conjunto solución de la ecuación $\sqrt{ }$ ?

La mayoría de los estudiantes del curso aplicaron correctamente el algoritmo diseñado para obtener la solución de la ecuación.

Ecuaciones de segundo grado. Para tratar ecuaciones de este tipo, se conocen fundamentalmente, los siguientes procedimientos:

- Aplicación de la formula cuadrática,

- Factorización

- Completación de cuadrados.

Las dos primeras estrategias son ampliamente conocidas por los estudiantes, sin embargo no reconocen la tercera como un procedimiento estándar por lo cual después de realizar algunas actividades para lograr competencia en esta última y en la búsqueda de procedimientos alternativos se propuso la siguiente actividad:

¿Es posible diseñar un procedimiento "diferente" que permita resolver una ecuación de segundo grado?

En realidad, no se tuvieron respuestas satisfactorias en este sentido, por lo que se tuvo la necesidad de reorientar el trabajo, con la siguiente actividad:

Para resolver la ecuación dada aplique la fórmula "incorrecta"

$\sqrt{ } /$

Una vez que se obtengan los valores correspondientes, piense en los siguientes interrogantes:

¿Se obtuvieron los valores correctos de las raíces?

Si esto no es así, ¿Qué debe realizar para obtener las soluciones correctas? 
Después de un análisis detenido y de las orientaciones correspondientes algunos estudiantes fueron capaces de concluir que al aplicar la fórmula sugerida se obtenían los inversos multiplicativos de las raíces, sin embargo no dieron razón del por qué esto debe ser así.

\section{Logros y dificultades evidenciadas}

Logros. Se recordaron los algoritmos usuales para resolver ecuaciones con radicales y cuadráticas y al proponer actividades diferentes a las rutinarias, los estudiantes trabajaron con mayor interés y se evidenció cómo compartían sus ideas y cómo las exponían frente a los demás. Dado que las respuestas iniciales a las actividades propuestas no fueron necesariamente correctas, se propició el análisis y la discusión del trabajo realizado en los grupos y del porqué en primera instancia no se había logrado la solución correcta.

Aunque en anteriores ocasiones se habían propuesto tareas con un mayor grado de dificultad e individualmente algunos de ellos habían entregado sus soluciones, estas no habían sido discutidas en el aula de clase de manera que fue la primera vez que se les dio el espacio para que ellos discutieran en grupo con lo cual el docente y los compañeros conocían las ideas que tenían y qué podían compartir y aportar a los demás grupos de trabajo.

Dificultades. La experiencia requirió gran dedicación por parte del docente en el diseño de las actividades ya que los procedimientos implementados no se encuentran en los libros de texto, pero lo más difícil fue lograr el compromiso de los estudiantes puesto que inicialmente, algunos de ellos, mostraban indiferencia por las actividades. Los estudiantes percibieron dificultades de tipo teórico ya el procedimiento diseñado para resolver ecuaciones con radicales no se puede aplicar a todo tipo de estas ecuaciones, en contraste con el algoritmo usual. El docente observó falta de análisis, en la resolución de las ecuaciones de segundo grado dado que aunque fueron capaces, con la orientación del profesor, de obtener las soluciones de la ecuación, no lograron explicar teóricamente porque el método debe dar siempre las raíces correctas, si

\section{Reflexión final}

Las actividades realizadas con los estudiantes fueron enriquecedoras para generar en ellos nuevos conocimientos y para fomentar el trabajo en grupo. Las diversas ideas que cada grupo discutió y que fueron compartidas con sus compañeros generó un ambiente de trabajo propicio para "hacer matemáticas", ya que alrededor de esta propuesta surgió una diversidad de conceptos y procedimientos que algunos de ellos aplican para llegar a resolver una situación dada.

Por otro lado, el tiempo invertido en el desarrollo de estas actividades es demasiado y aunque generan ambientes de aprendizaje que pretenden que los estudiantes exploren, interpreten, argumenten, propongan alternativas y cuestionen la práctica educativa, es necesario realizar un equilibrio de ellas ya que es posible enfatizar demasiado en estas y no alcanzar a tratar temas necesarios para el desarrollo de cursos posteriores.

Por último, vale la pena resaltar que a partir de este trabajo se han generado nuevas actividades que propenden por la participación y la construcción colectiva en el aula, tales como: construcción de gráficas y fórmulas para generar números primos.

\section{Referencias}

Chávez, H., Castañeda, N., Gómez, M., Joya, A., Chizner, J. \& Gómez, M. (2010). Hipertexto Matemáticas 9. Bogotá, Colombia: Santillana.

Ministerio de Educación Nacional. (1998). Lineamientos Curriculares de Matemáticas. Serie Lineamientos. Áreas Obligatorias y Fundamentales. Bogotá, Colombia: Cooperativa Editorial Magisterio.

Ministerio de Educación Nacional. (2006). Estándares Básicos de Competencias en Matemáticas. Bogotá, Colombia: Imprenta Nacional de Colombia.

Skovsmose, O. (2000). Escenarios de investigación. Revista EMA, 6 (1), 3-26. 